\title{
15-Lipoxygenase-1 re-expression in colorectal cancer alters endothelial cell features through enhanced expression of TSP-1 and ICAM-1
}

\author{
Sinem Tunçer ${ }^{\mathrm{a}}$, Ayşe Gökçe Keşküş ${ }^{\mathrm{b}}$, Melis Çolakoğlu ${ }^{\mathrm{a}}$, Ismail Çimen ${ }^{\mathrm{a}, 1}$, Caner Yener ${ }^{\mathrm{a}}$, \\ Özlen Konu ${ }^{\mathrm{b}}$, Sreeparna Banerjee ${ }^{\mathrm{a}, *}$ \\ ${ }^{a}$ Department of Biological Sciences, Orta Dogu Teknik Universitesi (ODTU/METU), Ankara 06800, Turkey \\ ${ }^{\mathrm{b}}$ Department of Molecular Biology and Genetics, Bilkent Universitesi, Ankara 06800, Turkey
}

\section{A R T I C L E I N F O}

\section{Keywords:}

15-LOX-1

Colorectal cancer

Angiogenesis

ICAM-1

Thrombospondin-1

\begin{abstract}
A B S T R A C T
15-lipoxygenase-1 (15-LOX-1) oxygenates linoleic acid to 13(S)-hydroxyoctadecadienoic acid (HODE). The enzyme is widely suppressed in different cancers and its re-expression has tumor suppressive effects. 15-LOX-1 has been shown to inhibit neoangiogenesis in colorectal cancer (CRC); in the present study we confirm this phenomenon and describe the mechanistic basis. We show that re-expression of 15-LOX-1 in CRC cell lines resulted in decreased transcriptional activity of HIF1 $\alpha$ and reduced the expression and secretion of VEGF in both normoxic and hypoxic conditions. Conditioned medium (CM) was obtained from CRC or prostate cancer cell lines re-expressing 15-LOX-1 (15-LOX-1CM). 15-LOX-1CM treated aortic rings from 6-week old C57BL/6 mice showed significantly less vessel sprouting and more organized structure of vascular network. Human umbilical vein endothelial cells (HUVECs) incubated with 15-LOX-1CM showed reduced motility, enhanced expression of intercellular cell adhesion molecule (ICAM-1) and reduced tube formation but no change in proliferation or cellcycle distribution. HUVECs incubated with 13(S)-HODE partially phenocopied the effects of 15-LOX-1CM, i.e., showed reduced motility and enhanced expression of ICAM-1, but did not reduce tube formation, implying the importance of additional factors. Therefore, a Proteome Profiler Angiogenesis Array was carried out, which showed that Thrombospondin-1 (TSP-1), a matrix glycoprotein known to strongly inhibit neovascularization, was expressed significantly more in HUVECs incubated with 15-LOX-1CM. TSP-1 blockage in HUVECs reduced the expression of ICAM-1 and enhanced cell motility, thereby providing a mechanism for reduced angiogenesis. The anti-angiogenic effects of 15-LOX-1 through enhanced expressions of ICAM-1 and TSP-1 are novel findings and should be explored further to develop therapeutic options.
\end{abstract}

\section{Introduction}

15-LOX-1 is a non-heme dioxygenase that preferentially metabolizes the essential polyunsaturated fatty acid (PUFA) linoleic acid into 13$(S)$-hydroxyeicosatetraenoic acid (13(S)-HODE). 15-LOX-1 has been shown to be universally lost in many different cancer cell lines [1], primarily through epigenetic mechanisms [2]. Re-expression of the gene has been shown to ameliorate a number of hallmarks of cancer [3-5].

Angiogenesis is a complex multistep process involving degradation of the extracellular matrix, migration and proliferation of endothelial cells (EC) and their reorganization into tube like structures. In adults, blood vessels are generally quiescent; ECs, however, are highly plastic cells that can respond to different angiogenic cues to initiate new blood vessel formation, thereby playing a crucial role in tumor growth and metastasis [6,7]. Tumor associated blood vessels are generally abnormal in both structure and function. The sprouting is chaotic; the ECs lack the typical cobblestone-like appearance and perfuse the tumor in a disorderly manner [7]. Previous studies have indicated that expression of 15-LOX-1 in prostate cancer (PCa) resulted in a pro-angiogenic effect [8]. On the other hand, anti-angiogenic effects of 15-LOX-1 have also been documented in rabbit skeletal muscle, rabbit eyes and mouse retinal epithelia [9-11]. Re-expression of 15-LOX-1 in CRC cell lines was shown to reduce the transcript levels of vascular endothelial growth factor-A (VEGF-A) and reduce the stability of the transcription factor hypoxia induced factor1 $\alpha$ (HIF1 $\alpha)$ [12].

In this study, we have aimed to mechanistically understand how 15LOX-1 expression in cancer cell leads to reduced angiogenesis. For this, we have expressed 15-LOX-1 in two CRC cell lines, HCT-116 and SW480 , and a prostate cancer cell line LNCaP. We observed that 15-LOX-1

\footnotetext{
* Corresponding author.

E-mail address: banerjee@metu.edu.tr (S. Banerjee).

${ }^{1}$ Current address: Institute for Cardiovascular Prevention, Ludwig Maximilian University, Munich 80 336, Germany.
} 
over-expression in the cancer cell lines resulted in reduced secretion of angiogenic factors. Conditioned medium (CM) from these cells caused several alterations in ECs, primarily in EC motility rather than proliferation. This was corroborated by enhanced expression in ECs of intercellular cell adhesion molecule (ICAM-1) that is known to enhance the formation of EC polarity and proper directionality of movement [13] and the antiangiogenic protein thrombospondin-1(TSP-1) that inhibits neovascularization through reduced VEGF-A activity, enhanced apoptosis of ECs, inhibition of EC migration, and suppression of nitric oxide signaling [14].

\section{Materials and methods}

\subsection{Cell culture, transfections and treatments}

Human CRC cell line SW-480, LNCaP and the human vascular endothelial cells (HUVECs) were obtained from ATCC (Middlesex, England). HCT-116 cells were obtained from DSMZ (Braunschweig, Germany). The epithelial cells were grown in RPMI-1640 (Biochrom, Berlin, Germany) supplemented with 10\% fetal bovine serum (FBS) (Thermo Scientific, Waltham MA, USA), $2 \mathrm{mM}$ L-glutamine and $1 \%$ penicillin/streptomycin. HUVECs were grown on gelatin-coated plates with EBM Endothelial Cell Medium (ECM) Bullet Kit (Lonza, Basel, Switzerland) and used up to the 5th passage. All cell lines used were authenticated at the University of Arizona, Genetics Core Facility, Arizona, USA.

The stable transfection of HCT-116 with a 15-LOX-1-pcDNA3.1 vector for the expression of the human ALOX15 gene (kind gift from Dr. Uddhav Kelavkar [8] or empty vector (EV) has been described by our group previously $[3,4,15]$. Where indicated, two individual monoclones stably expressing 15-LOX-1 were used. All stably transfected cells were maintained in $125 \mu \mathrm{g} / \mathrm{ml}$ zeocin as the selection antibiotic. For the transient expression of 15-LOX-1 in SW-480 or LNCaP cells, $7.5 \times 10^{5}$ cells per well were seeded in 6-well plates and transfected for $24 \mathrm{~h}$ with X-tremeGENE HP (Roche, Mannheim, Germany) at a 1:1 ratio. The medium was changed and the cells were then incubated for a further $48 \mathrm{~h}$ after which the cells and the conditioned medium (CM) were collected. Each transfection was confirmed for 15-LOX-1 expression by western blot. To collect CM from HCT-116 cells stably transfected with the 15-LOX-1 plasmid, cells at $70-80 \%$ confluency were grown in zeocin-free medium for $72 \mathrm{~h}$, the medium was collected, centrifuged at $100 \times g$ for $10 \mathrm{~min}$, aliquoted and stored at $-80^{\circ} \mathrm{C}$ until use. To grow cells under hypoxia, a Hypoxia Incubator Chamber (Stemcell Technologies, Canada) was filled with a premixed gas containing $1 \% \mathrm{O}_{2}, 5 \%$ $\mathrm{CO}_{2}$ and $94 \% \mathrm{~N}_{2}$ (confirmed with an oxygen sensor) and sealed. Before starting the experiment, the cell culture medium was degassed overnight. Flasks containing the degassed medium were placed in the chamber, which was refluxed with the premixed gas after $1.5 \mathrm{~h}$ and incubated for a total of $6 \mathrm{~h}$. Preliminary experiments showed that there was minimal cell death and a robust induction of VEGF-A after $6 \mathrm{~h}$ in hypoxia. In each case, a parallel experiment was conducted under normoxia for comparison.

Where indicated, HCT-116 cells stably transfected with a 15-LOX-1 vector or the corresponding EV were treated with $10 \mu \mathrm{M}$ of the 15-LOX1 inhibitor PD146178, HUVECs were treated with $25 \mu \mathrm{M}$ 13(S)-HODE (Cayman Chemical, USA) or $100 \mathrm{ng} / \mathrm{ml}$ VEGF-A (Merck, Germany) for $24 \mathrm{~h}$ in complete medium.

\subsection{5-LOX-1 activity measurement}

13(S)-HODE, the metabolic product of 15-LOX-1, was measured with a competitive immunoassay kit (Enzo Life Sciences, USA) according to the manufacturer's instructions. Briefly, $2 \times 10^{6}$ of HCT-116 cells stably transfected with 15-LOX-1 or EV were harvested, lysed, and acidified with $0.2 \mathrm{~N} \mathrm{HCl}$. Organic phase from the samples was extracted with water-saturated ethyl acetate. The extracts were dried completely using benchtop Freeze Dry System (FreeZone 6 Liter, Labconco, USA). Dried samples were dissolved in $25 \mu \mathrm{l}$ ethanol, followed by 1:10 dilution with Assay Buffer. Samples were then applied to goat anti-rabbit IgG microtiter plate, and the level of 13(S)-HODE was measured colorimetrically using a microplate spectrophotometer (Thermo Scientific, USA) at $405 \mathrm{~nm}$ with correction at $580 \mathrm{~nm}$. The 13(S)-HODE concentrations in the samples were determined from a standard curve.

\subsection{RNA isolation, cDNA synthesis and RT-qPCR assays}

Total RNA isolation was carried out by using RNeasy RNA Extraction Kit (Qiagen, Hilden, Germany) according to the manufacturer's guidelines. cDNA synthesis from DNAse I (Thermo Scientific) treated mRNA was carried out with $1 \mu \mathrm{g}$ of the RNA using a RevertAid First Strand cDNA Synthesis Kit (Thermo Scientific).

RT-qPCR reactions were carried out in a Rotor GeneQ 6000 (Qiagen) using standard techniques. Fold changes were calculated with respect to the internal control ( $\beta$-Actin) using the Pfaffl method [16]. MIQE guidelines were followed in the RT-qPCR reactions [17]. The primer sequences are shown in Supplementary Table 1.

\subsection{VEGF secretion assay}

The secreted VEGF- $\mathrm{A}_{165}$ levels in cell culture supernatants was measured with the Quantikine Human VEGF Immunoassay kit (R \& D Systems, Minneapolis, MN, USA) according to the manufacturer's protocol.

\subsection{Western blotting}

Proteins $(20-50 \mu \mathrm{g})$ were separated in $10 \%$ SDS-PAGE gels and transferred to PVDF membranes using standard techniques. The antibodies used are shown in Supplementary Table 2. Bands were visualized with the Clarity ECL Substrate (Bio-Rad, Hercules, CA, USA) and ChemiDoc MP Imaging System (Bio-Rad).

\subsection{Luciferase assay}

HCT-116 cells stably transfected with 15-LOX-1 or EV were plated in 48-well plates $\left(5 \times 10^{4}\right.$ cells/well) and allowed to attach overnight. After transfection with $300 \mathrm{ng}$ of pGL3-5xHRE-VEGF-Luc (generous gift from Dr. Ilias Mylonis) [18] and pRL-TK Renilla (Promega, Madison, WI, USA) (Firefly:Renilla ratio of 250:1) using X-tremeGENE HP (1:2 plasmid:transfection reagent ratio), the cells were incubated under hypoxia or normoxia for an additional $6 \mathrm{~h}$. The luciferase assay was conducted with the Dual-Luciferase Assay kit (Promega) according to the manufacturer's instructions.

\subsection{Mouse aorta ring assay}

To determine the effect of 15-LOX-1CM on the sprouting ability of endothelial cells, an ex vivo mouse aorta ring assay was carried out as described previously [19]. The study was approved by the animal ethics committee of Bilkent University (Decision no: 2014/36). Institutional and national guidelines for the handling and care of animals were followed. Aortas were removed from 6 to 8 weeks old C57BL/ 6 mice, cut into $1.0 \mathrm{~mm}$ rings, embedded in Matrigel and incubated in $500 \mu \mathrm{l}$ of a 1:1 mixture of CM and OptiMEM containing 2.5\% FBS and 1\% Pen/ Strep at $37^{\circ} \mathrm{C}$ for 9 days. The medium was changed every other day. The images acquired on the 9th day were analyzed using WimSprout (Wimasis, Munich, Germany). Rings incubated in medium with $20 \%$ FBS and $2.5 \%$ FBS served as positive and negative controls, respectively. The experiment was repeated three times independently and aortas from three different mice were used for each experiment. 


\subsection{HUVEC tube formation assay}

The tube formation assay was carried out using HUVECs embedded in Matrigel in a 1:1 ratio of CM and ECM as described previously [20]. Where indicated, HUVECs were treated with $25 \mu \mathrm{M} 13(S)$-HODE in 1:1 ratio of RPMI-1640 complete growth medium and ECM. Cells were incubated for $6 \mathrm{~h}$ and tubes were photographed with an inverted microscope (Olympus, Hamburg, Germany) or JuLi Smart Fluorescence Cell Imager. The results were analyzed using WimTube (Wimasis).

\subsection{HUVEC proliferation and cell cycle assay}

Proliferation of HUVECs incubated in a 1:1 ratio of CM and ECM was determined by an MTT assay. Briefly, $5 \times 10^{3}$ HUVECs were plated in each well of a 96-well plate and allowed to attach overnight. The MTT assay was conducted according to standard protocols. Measurements were carried out at $570 \mathrm{~nm}$ in a plate reader (Thermo Scientific).

For cell cycle analysis, HUVECs at 50\% confluency were treated with a 1:1 ratio of CM and ECM for $24 \mathrm{~h}$. The cells were collected, counted and $5 \times 10^{5}$ cells were fixed with $70 \%$ ethanol overnight at $-20{ }^{\circ} \mathrm{C}$. The cells were washed with $1 \times$ PBS and incubated in a staining solution containing $0.1 \%$ Triton-X (Applichem), $0.2 \mathrm{mg} / \mathrm{ml}$ RNase (Thermo Scientific) and $15 \mu \mathrm{g} / \mathrm{ml}$ Propidium iodide (Sigma, Taufikirchen, Germany) for $30 \mathrm{~min}$ in the dark at room temperature. The cell cycle distribution was obtained from a flow cytometer (BD Biosciences, Ann Arbor, MI, USA) and analyzed with the BD Accuri C6 software.

\subsection{In vitro wound healing assay}

Scratches were made with sterile $200 \mu 1$ pipette tips in $100 \%$ confluent HUVECs incubated with 1:1 ratio of CM and ECM or $25 \mu \mathrm{M}$ 13(S)-HODE containing RPMI-1640 complete growth medium and ECM for $15 \mathrm{~h}$. Cells were photographed with a JuLi Smart Fluorescence Cell Imager at $3 \mathrm{~h}$ intervals, a total of $9 \mathrm{~h}$. Where indicated, followed by $18 \mathrm{~h}$ incubation with 1:1 ratio of CM and ECM, blocking experiments for $6 \mathrm{~h}$ with a TSP-1 or isotype specific IgG antibody were carried out.

\subsection{Proteome profiler human angiogenesis array}

HUVECs were incubated with a 1:1 ratio of CM and ECM for $24 \mathrm{~h}$ and lysed in a buffer containing 1\% NP-40, 20 mM Tris- $\mathrm{HCl}$ (pH 8.0), $137 \mathrm{mM} \mathrm{NaCl}, 10 \%$ glycerol, $2 \mathrm{mM}$ EDTA and $1 \times$ protease inhibitor cocktail (Roche). $200 \mu \mathrm{g}$ of the lysate was used per membrane of a Proteome Profiler Array, Human Angiogenesis Array Kit (R \& D Systems). Three independent angiogenesis arrays with three independent protein sets from two lots of HUVECs were conducted. After visualizing the spots, the signals were measured with a Protein Array Analyzer plugin for ImageJ (https://imagej.nih.gov/ij/). Intensity files obtained from Image $J$ were then uploaded to an in-house MATLAB code (MATLAB R2016a and Statistics Toolbox 11.0, The MathWorks, Inc., Natick, MA, USA, code available upon request). Details on normalizations methods are available in Supplementary Fig. 6.

\subsection{Bioinformatic data analyses}

CEL files of GSE43133 were downloaded from NCBI GEO database [21]. Samples labeled GSM1057218-23 belonging to CD47 wild-type or null lung ECs of mice (C57Bl/6) were used in further analyses. gcrma R package from bioconductor.org was used to perform robust multiarray average (RMA) normalization [22]. For differential gene expression analysis, limma package of bioconductor was used [23]. Genes differentially expressed at $p<0.05$ were determined; from these, genes that belonged to the GO:0016477 Cell Migration term and GO:0007155 Cell Adhesion term were downloaded from the BioMart facility of Ensembl (release 84), AmiGO2 (version 2.5.5) and PANTHER (11.1) databases [24-26]. Only those genes appeared in all three databases were used for further analyses; Fisher's Exact test was performed for the significance analysis of GO term enrichment (Matlab).

CEL files of GSE77199 datasets and the pre-processed data for GSE2177 were downloaded from the NCBI GEO database [27,28]. For GSE2177, data from GSM39408 (vehicle treated Human Microvascular Endothelial Cells, HMVECs)) and GSM39409 (HMVECs treated with DITSPa, a TSP mimetic peptide) were globally normalized and log transformed to estimate the log fold change. For GSE77199, samples labeled GSM2045928-GSM2045931 (healthy colon EC) and those labeled GSM2045932-GSM2045935 (CRC associated EC) were used. limma [23] package was used for normalization and paired differential expression analysis. We then performed principal components analysis (PCA) for detecting outlier pair(s); accordingly, GSM2045934 and the corresponding healthy sample, GSM2045930, that showed negative or no correlation with other subjects were removed. Genes differentially expressed at $p<0.05$ were determined. Genes altered in the opposite direction in GSE2177 and GSE77199 were used for identifying proteinprotein interactions (default setting) using STRING 10.0 [29,30]. Based on STRING we then identified enriched GO Terms in the largest connected component and the first five GO Terms were selected. If more than one term belonged to the same ancestor only the ancestor term was used. We plotted the network in Matlab using a color palette.

\subsection{Statistical analyses}

Results were expressed as the mean \pm standard error of mean (SEM). Data analyses and graphing were carried out with GraphPad Prism 6.0. Two-way ANOVA, Student's $t$-tests or limma were used for statistical analysis, and differences at $p \leq 0.05$ were considered significant. When more than a single factor was present a Two-way ANOVA also was applied to test for the main and interaction effects. Experiments were repeated independently at least three times with at least three technical replicates.

\section{Results}

3.1. 15-LOX-1 expression in cancer cell lines reduced VEGF expression and secretion

The cell lines used in this study did not express any 15-LOX-1 within the detection limits of a western blot. HCT-116 cells stably transfected (clones 1 and 2) and SW-480 and LNCaP cells transiently transfected with the 15-LOX-1 plasmid resulted in robust expression of the 15-LOX1 protein compared to the EV transfected control cells (Fig. 1A, Supplementary Fig. 1A, B). The enzymatic activity of 15-LOX-1 in stably transfected HCT-116 cells was confirmed by a 13(S)-HODE ELISA kit (Fig. 1B).

VEGF-A (commonly referred to as VEGF) is strongly implicated in angiogenesis [31]. We observed a significant reduction in mRNA expression of VEGF in 15-LOX-1 expressing HCT-116 (clones 1 and 2, Fig. 1C), SW-480 cells (Supplementary Figure Fig. 1C) and LNCaP cells (Supplementary Fig. 1D). Moreover, when the 15-LOX-1 expressing HCT-116 cells (clone 1) were treated with PD146176 (a 15-LOX-1 inhibitor that reduces the synthesis of 13(S)-HODE [3]), we observed a recovery of VEGF mRNA expression to a level comparable with the EV transfected cells (Fig. 1F). Angiogenesis is generally activated in a hypoxic tumor environment, which leads to the stabilization of transcription factors called hypoxia inducible factors (HIFs) that can then upregulate VEGF expression [7]. 15-LOX-1 mediated reduction in VEGF mRNA levels was retained even under hypoxia (Fig. 1D, Supplementary Fig. 1C). The reduced VEGF expression in 15-LOX-1 expressing cells was accompanied by a reduction in VEGF secretion under both hypoxic and normoxic conditions (Figs. 1E, Supplementary Fig. 1C, D). A luciferase construct containing 5 copies of the HIF1 $\alpha$ binding sequence in 
A

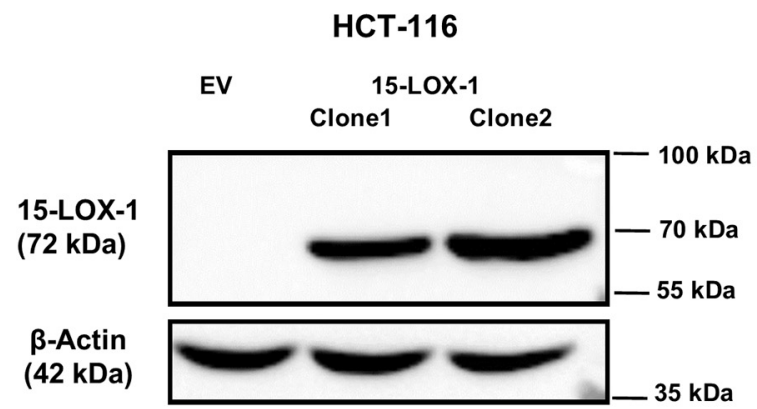

B

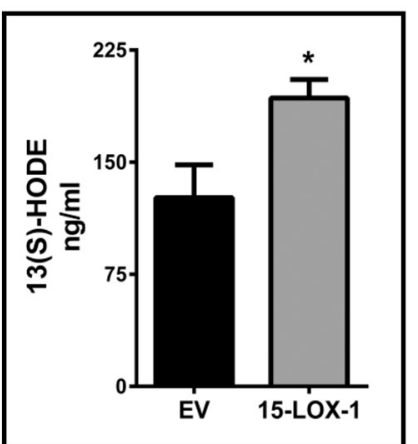

C

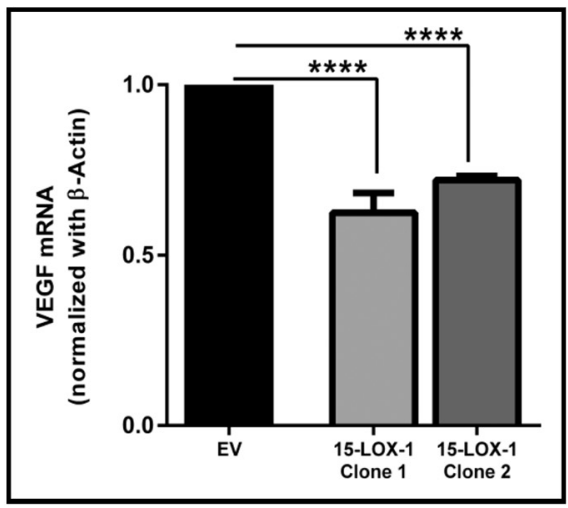

$\mathbf{F}$

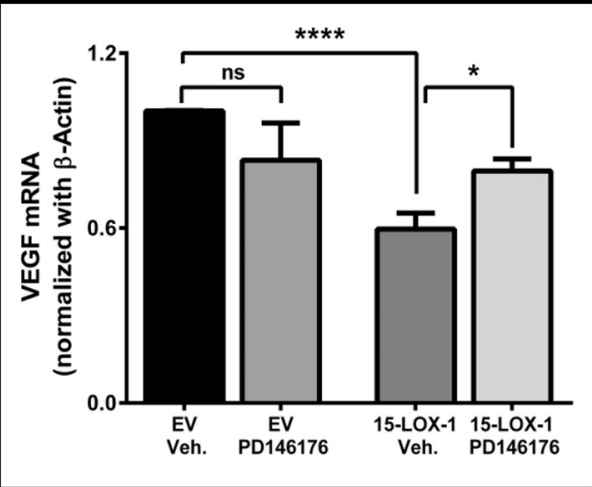

D

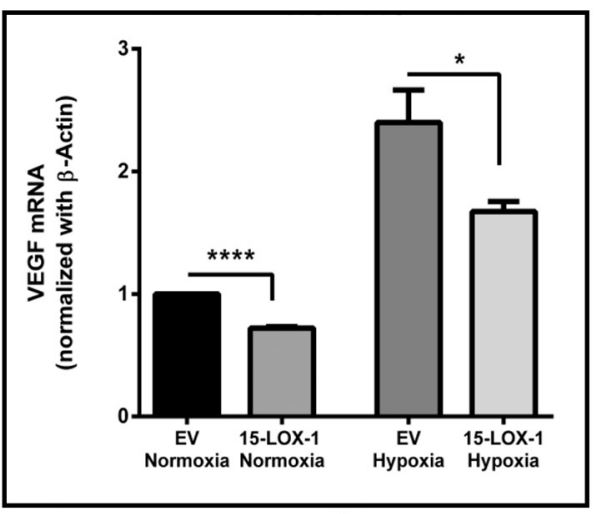

E

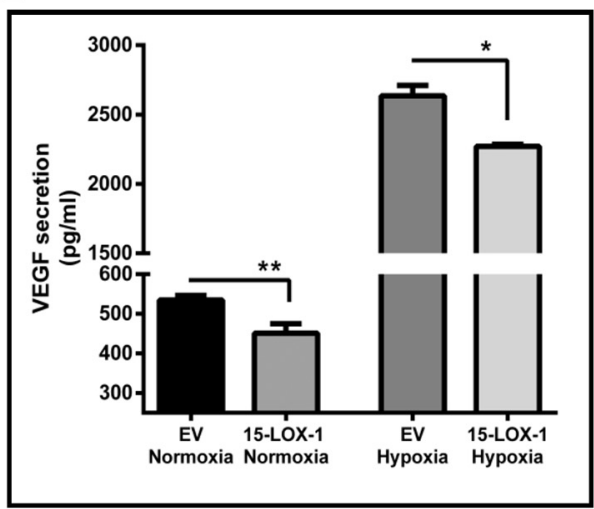

\section{G}

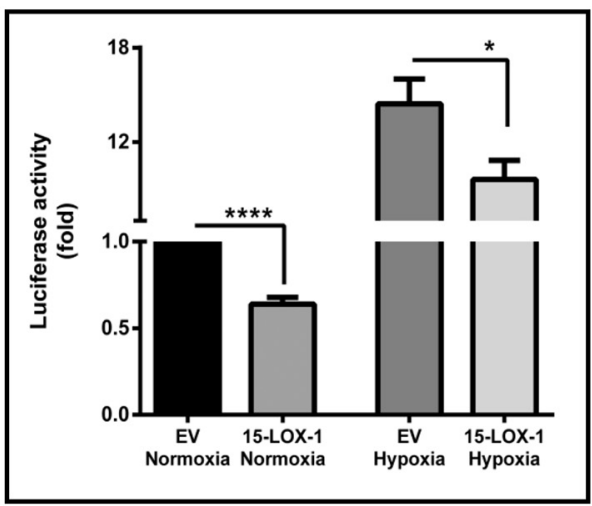

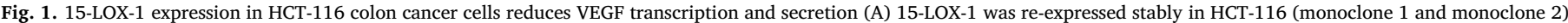

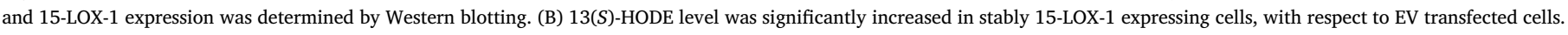

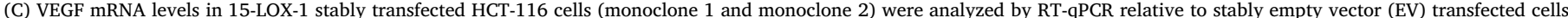

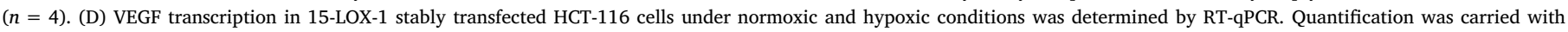

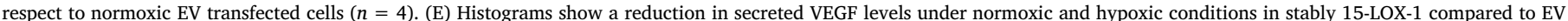

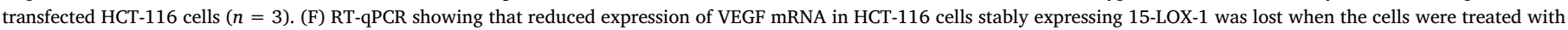

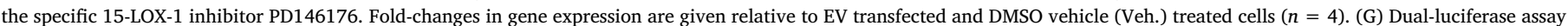

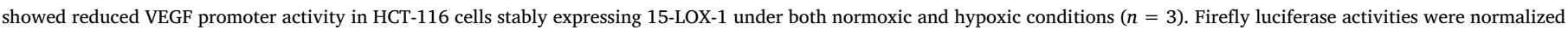

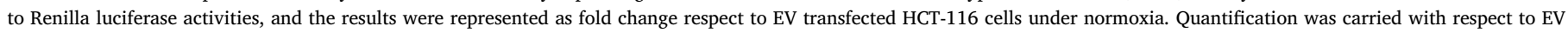
transfected cells. All results were analyzed with $t$-test $\left({ }^{*} p \leq 0.05,{ }^{* *} p \leq 0.01,{ }^{* * *} p \leq 0.001,{ }^{* * * *} p \leq 0.0001\right)$.

the VEGF promoter showed reduced activity in the 15-LOX-1 expressing cells in both hypoxic and normoxic conditions (Fig. 1G).

\subsection{Conditioned medium obtained from 15-LOX-1 expressing cells reduced angiogenic sprouting and tube formation}

Paracrine factors released from tumor cell can affect angiogenic signaling $[31,32]$. To examine this, we carried out an ex vivo aortic ring assay where, in the presence of appropriate signals, quiescent ECs in the aorta are activated to form organized sprouts. Aortas incubated with 15-LOX-1CM showed reduced sprouting and pseudocapillary tubes as well as lower sprout area and sprout length. The mean ring areas of the aortas incubated with the 15-LOX-1CM versus the EVCM were similar, indicating that the differences observed in sprouting were unrelated to the ring area (Fig. 2A).

We next incubated HUVECs in a 1:1 mixture of CM from stably 15LOX-1 transfected HCT-116 cells and ECM. Significantly lower tube length and total number of tubes along with fewer branches and loops were seen in HUVECs incubated with 15-LOX-1CM from HCT-116 cells compared to the control EVCM (Fig. 2B). These differences became even more pronounced when the HUVECs were incubated in CM collected after maintaining the cells under hypoxia for $6 \mathrm{~h}$. Similar changes 


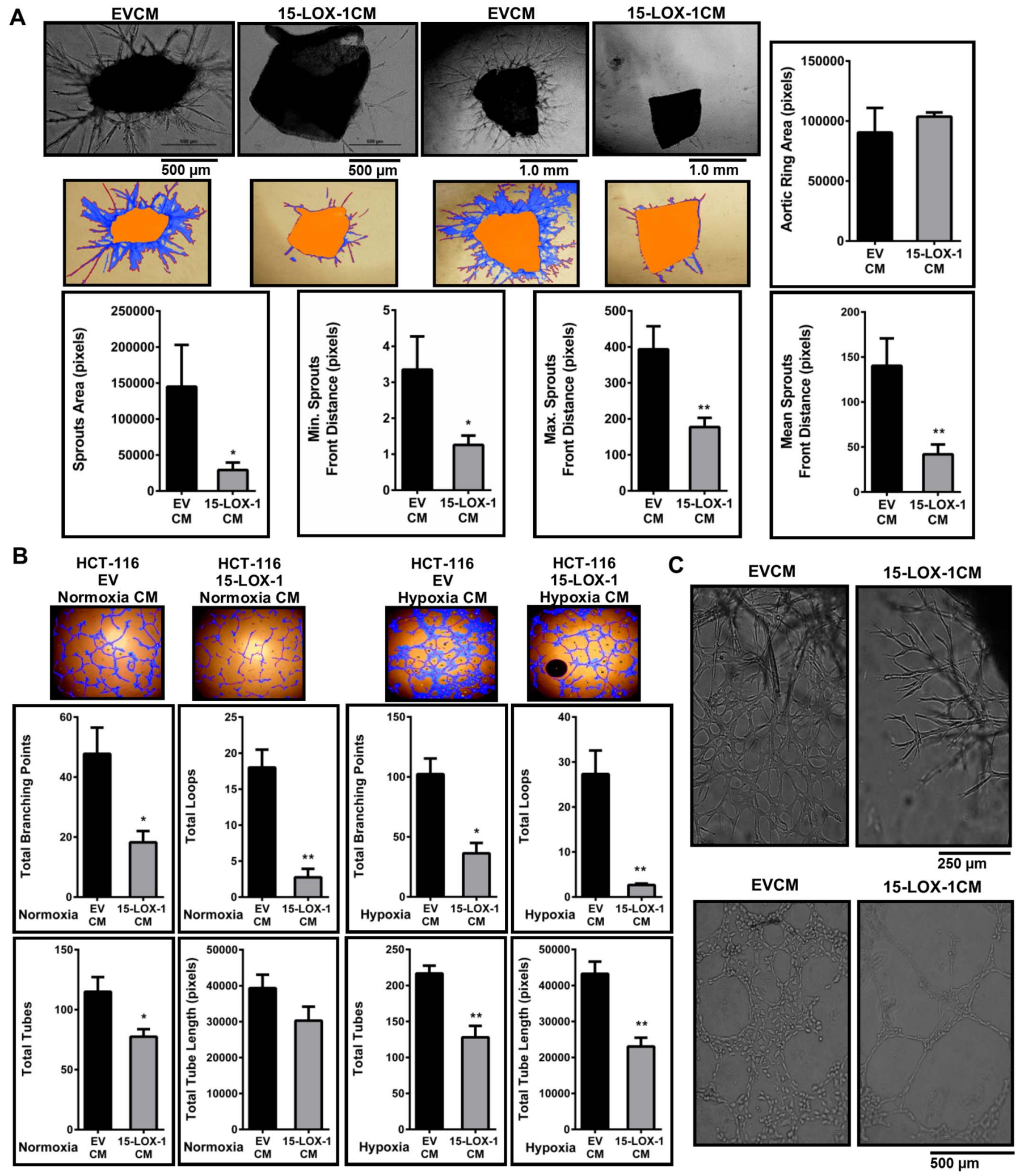

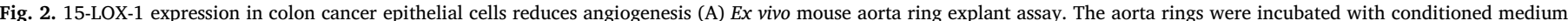

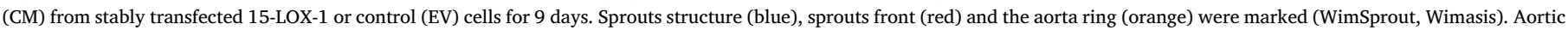

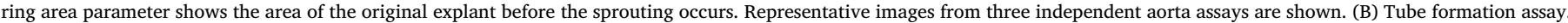

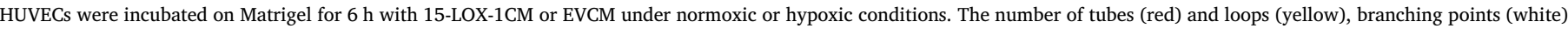

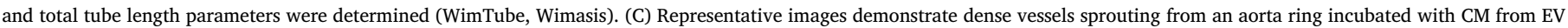

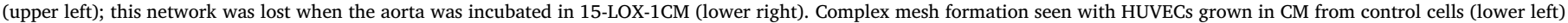

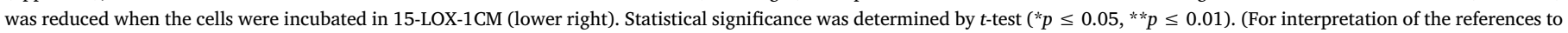
color in this figure legend, the reader is referred to the web version of this article.) 
were observed with CM obtained from SW-480 cells and LNCaP cells expressing 15-LOX-1 (Supplementary Fig. 2A and B).

Overall, we observed that secreted factors from 15-LOX-1 expressing cells resulted in a reduced ability of ECs to sprout and form tubes both ex vivo and in vitro. Moreover, mouse aorta rings incubated with EVCM showed a rather dense and chaotic network with irregular branching pattern, most likely representing collateral vessels [33]. This network was lost in the rings incubated with 15-LOX-1CM (Fig. 2C, upper panel). Additionally, complex mesh formation [36], which are large rounded structures with walls that are four to five cells thick were seen in the HUVECs incubated with EVCM (Fig. 2C, lower panel) but not in the HUVECs incubated with 15-LOX-1CM. These findings indicated the possibility that secreted factors in 15-LOX-1CM may result in the normalization of tumor vasculature. Tumor associated endothelial cells (TECs) have abnormal branching morphogenesis and sprout excessively [33]. We first established that tumor conditioned HUVECs could display TEC characteristics. We grew HUVECs in a 1:1 mixture of ECM and CM from wild-type HCT-116 cells and observed a 2-2.5 fold increase in the expression of Fibroblast Associated Protein- $\alpha$ (FAP) (Supplementary Fig. 3A), a well-established TEC marker [34,35]. We observed significant, albeit modest decreases in the expression of the TEC markers Protein Regulator of Cytokinesis 1 (PRC-1) [37] and ADAM Metallopeptidase Domain 23 (ADAM23) [34] in HUVECs incubated with 15-LOX-1CM compared to the control cells (Supplementary Fig. 3B).

\subsection{Conditioned medium obtained from 15-LOX-1 expressing cells reduced motility of HUVECs}

ECs were incubated in 15-LOX-1CM and analyzed for proliferation, cell cycle and motility. Interestingly, we did not observe any alteration in the proliferation of HUVECs incubated with 15-LOX-1CM from either HCT-116 or SW-480 cells, indicating that reduced tube formation was unrelated to proliferation (Fig. 3A). Corroborating these data, cell cycle analysis of HUVECs incubated with either 15-LOX-1CM or EVCM from
HCT-116 cells did not result in significant alterations in cell cycle stages (Supplementary Fig. 4). On the other hand, a scratch wound healing assay showed significantly decreased motility of HUVECs when the cells were incubated with 15-LOX-1CM from HCT-116 compared to EVCM (Fig. 3B). We next probed the cells for expression of cell adhesion markers and observed an increase in the expression of ICAM-1 (Fig. 3C, Supplementary Fig. 5 for 15-LOX-1CM from LNCaP cells) and a decrease in the expression of the mesenchymal marker Vimentin (Fig. 3C) in HUVECs treated with 15-LOX-1CM compared to the control EVCM treated cells. No change was observed in the expression of VCAM-1. These data indicate that $15-\mathrm{LOX}-1 \mathrm{CM}$ could reduce the motility of the cells, without affecting their proliferation or cell cycle distribution.

\subsection{Incubation of EC's with 13(S)-HODE phenocopied most, but not all features of 15-LOX-1CM treatment}

15-LOX-1 overexpression in cells leads to an enhanced production of 13(S)-HODE from the substrate linoleic acid. This bioactive lipid has previously been shown to affect endothelial cell characteristics in models of atherosclerosis [34] as well as cancer [12]. Incubation of HUVECs with a 1:1 mixture of ECM and complete RPMI-1640 containing $25 \mu \mathrm{M} 13(S)$-HODE led to reduced motility in a scratch wound assay (Fig. 4A) and remarkably enhanced expression of ICAM-1 (Fig. 4B), but did not lead to any alteration in tube formation (Fig. 4C). Therefore, additional inhibitory factors in the tumor microenvironment are likely to contribute to the reduced tube formation observed with 15LOX-1CM.

\subsection{Proteome profiler array analysis in HUVECs incubated with 15-LOX- $1 \mathrm{CM}$}

To define any additional factors that may contribute to reduced angiogenesis and potential vascular normalization observed with 15LOX-1CM, we examined the expression of 55 angiogenesis related proteins. A Proteome Profiler Array was used with protein lysates from
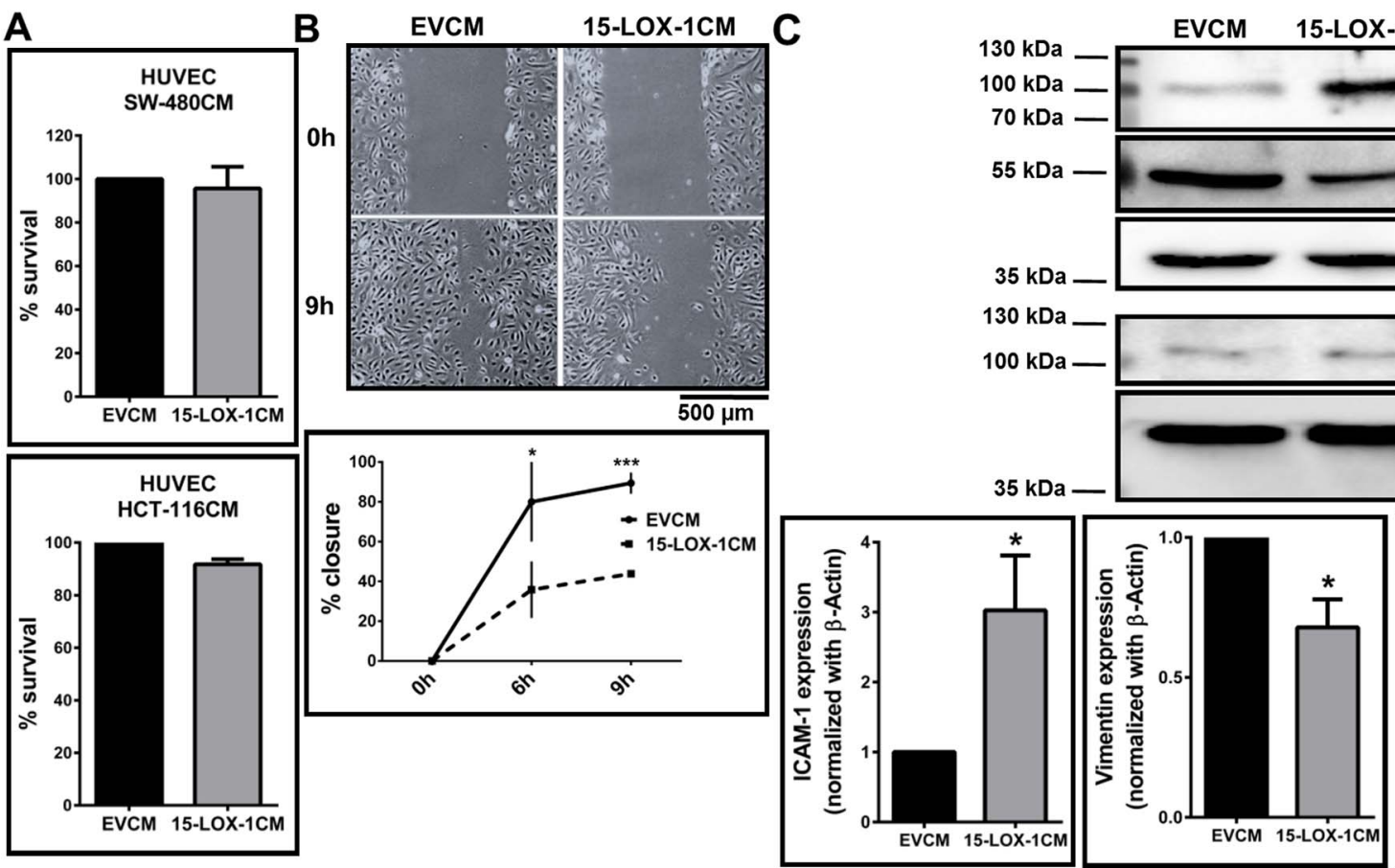

ICAM-1

(85-110 kDa)

\section{Vimentin}

(57 kDa)

\section{$\beta$-Actin}

(42 kDa)

VCAM-1

(110 kDa)

$\beta$-Actin

(42 kDa)
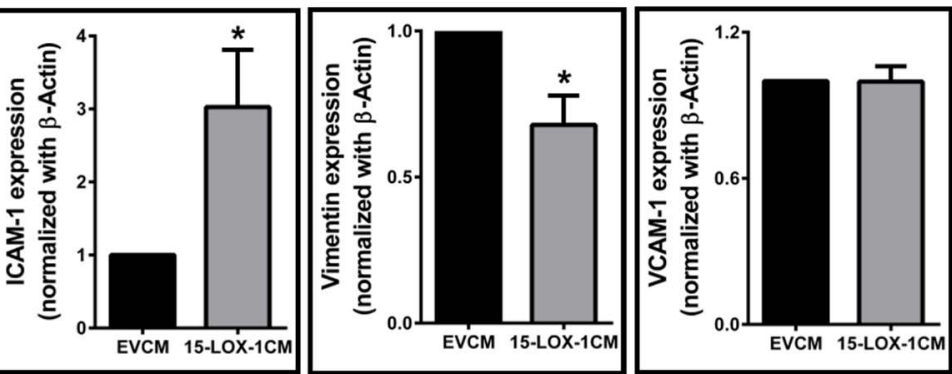

Fig. 3. Effect of conditioned medium (CM) from 15-LOX-1 expressing colon cancer epithelial cells on endothelial cell proliferation and motility (A) HUVECs were incubated with CM from HCT-116 stably expressing 15-LOX-1 or transiently transfected SW-480 cells for $24 \mathrm{~h}$, and viability of HUVECs was determined by MTT assay. Cell survival is represented as percent respect to EVCM (control) treated cells. (B) Scratch assay shows the time dependent migration capacities of HUVECs treated with CM obtained from stably 15-LOX-1 or EV expressing HCT-116 cells. Two-way ANOVA was used to compare the means ( $\left.{ }^{*} p \leq 0.05, * * * p \leq 0.001\right)$. (C) Western blot showing the expression of ICAM-1, VCAM-1 and vimentin in HUVECs treated with 15-LOX-1CM or EVCM. Statistical significance was determined by $t$-test $(" p \leq 0.05)$. 

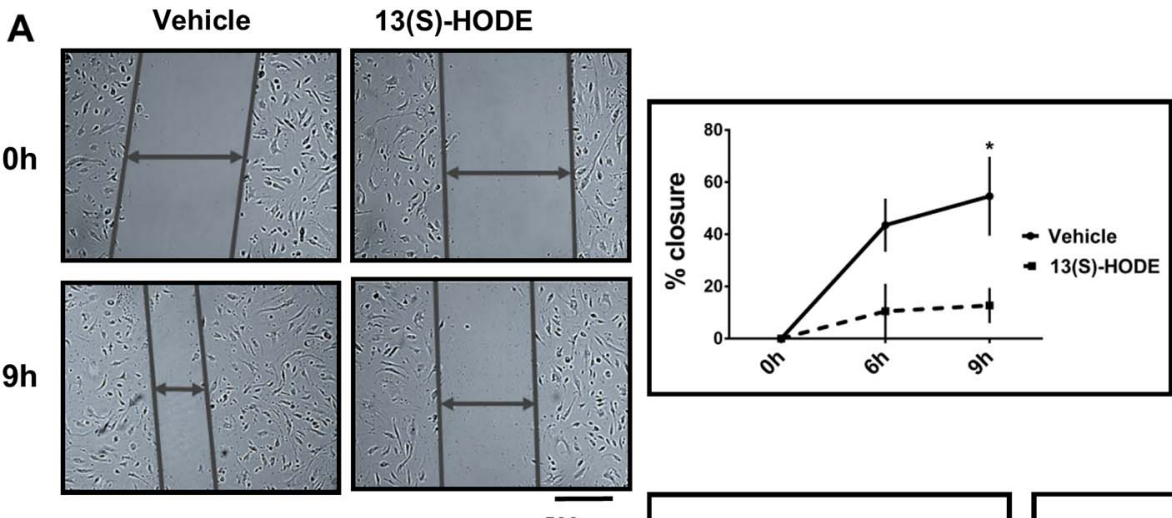

B
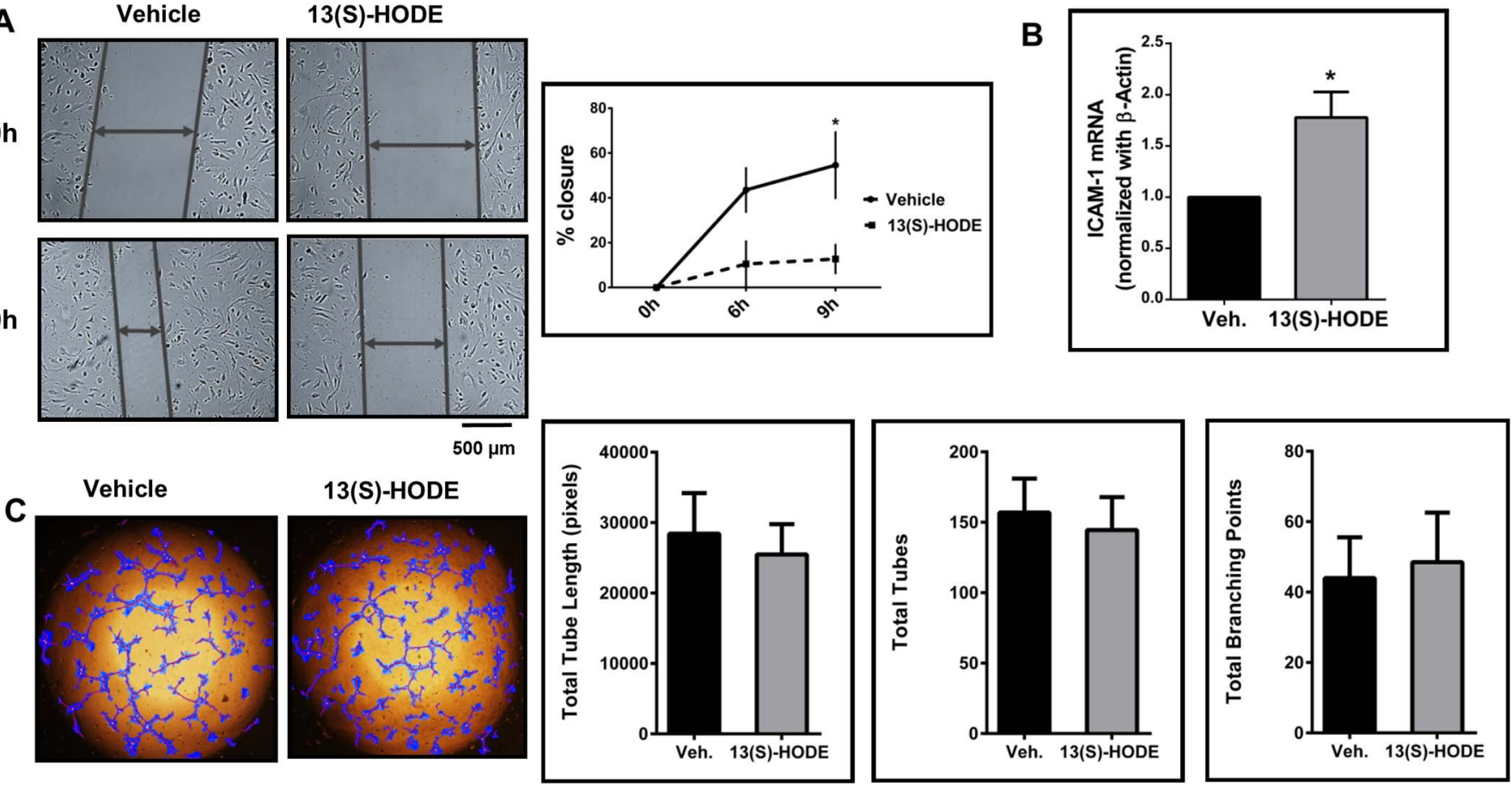

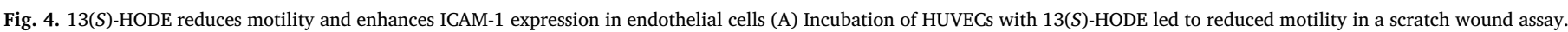

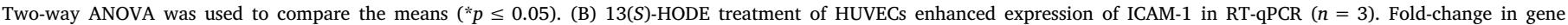

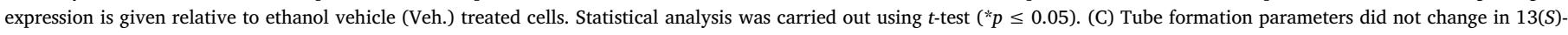
HODE treated HUVECs.

HUVECs incubated with 15-LOX-1CM from HCT-116 cells or EVCM (Supplementary Fig. 6A). Our analyses revealed that correlation between the duplicate spots was very strong for all arrays indicating the high quality of the experiments (Supplementary Fig. 6B). When unnormalized (Supplementary Fig. 6C), and even after global normalization (Supplementary Fig. 6D) data distribution within each batch of the array sets (experiment $v$ s. control for experiments 1-3) were more similar to each other than in between sets. Batch correction performed by subtracting the control spot value from that of the corresponding experiment spot normalized the arrays further and made them more comparable (Supplementary Fig. 6E). A significant increase in the protein levels of Thrombospondin-1 (TSP-1) was observed (Fig. 5A; $t$ test $p=0.044$ ) in HUVECs incubated with 15-LOX-1CM. The significant increase was further confirmed at both the protein and mRNA levels from HUVECs incubated with 15-LOX-1CM compared to EVCM (Fig. 5B).

To understand whether the altered expression of TSP-1 in HUVECs treated with 15-LOX-1CM was due to the enhanced secretion of 13(S)HODE, we treated HUVECs with $25 \mu \mathrm{M}$ 13(S)-HODE, but did not observe a significant change in the expression of TSP-1 (Fig. 5C). On the other hand, treatment of HUVECs with $100 \mathrm{ng} / \mathrm{ml}$ recombinant human VEGF resulted in a significant decrease in the expression of TSP-1 (Fig. 5D). It is therefore likely that the enhanced expression of TSP-1 observed in HUVECs treated with 15-LOX-1CM was due to the reduced secretion of VEGF by the 15-LOX-1 re-expressing cancer cells.

\subsection{Signaling mechanism associated with decreased motility of HUVECs incubated with 15-LOX-1CM}

We next aimed to address the signaling mechanism resulting from enhanced levels of TSP-1 in endothelial cells incubated with CM from 15-LOX-1 expressing cells. TSP-1 primarily binds to CD47 and CD36 as the cognate receptors for further downstream signaling [35]. Large vessel ECs express little or no CD36 [36]; we have observed considerably lower expression of CD36 when compared to CD47 in the
HUVECs used in the current study (Supplementary Fig. 7A). No difference in the expression of CD47 was observed when the HUVECs were treated with either 15-LOX-1CM or EVCM (Supplementary Fig. 7B). Additionally, TSP-1, by binding to CD36, can inhibit signaling through VEGFR2 [37]. We observed no change in the phosphorylation of VEGFR2 (Tyr 1175) in HUVECs treated with 15-LOX-1CM (Supplementary Fig. 7C). It is thus likely that TSP-1 mediates its effects via CD47 in the model examined in the current study. Based on this, we explored a publicly available microarray data from ECs of a CD47 null mouse model (GSE43133). The expression of $20.6 \%$ and $20.8 \%$ of all genes associated with GO terms migration (Fisher Exact test $p=3.38 \mathrm{e}-04$ ) and adhesion (Fisher Exact test $p=1.29 \mathrm{e}-08$ ), respectively, were altered in these cells (Fig. 6A) compared to $16 \%$ of altered genes in the entire dataset. In mice, ICAM-1 was significantly downregulated with the loss of CD47 in the ECs (Fig. $6 \mathrm{~B} ; p=0.0008$ ). When we examined the levels of ICAM-1 in the HUVECs incubated with 15-LOX-1CM for $24 \mathrm{~h}$, we observed a significant upregulation in ICAM1 (Fig. 6C; $p \leq 0.05$ ). More importantly, when these cells were also incubated with a TSP-1 blocking antibody for $6 \mathrm{~h}$, we observed a significant decrease in the levels of ICAM-1 at both mRNA and protein levels (Fig. 6C) indicating that the increase in ICAM-1 was also mediated through TSP-1 signaling. To functionally determine whether TSP-1 blocking could affect EC motility, we carried out a scratch wound healing assay where TSP-1 mediated signaling was inhibited with a blocking antibody or an isotype specific IgG antibody. In the HUVECs incubated with EVCM, blocking of TSP-1 resulted in a significantly greater motility of the cells compared to HUVECs incubated with IgG (Fig. 6D). Of note, recovery in motility to a similar extent was also seen in the HUVECs incubated with 15-LOX-1CM and TSP-1 blocking antibody ( $p=0.2592$, two-way ANOVA with anovan in Matlab). The overall motility of HUVECs incubated in 15-LOX-1CM was low indicating that factors in addition to TSP- 1 in the CM may also have contributed to the reduced motility seen in these cells.

HUVECs incubated with 15-LOX-1CM showed a potential vascular normalization (Fig. 2C). To determine whether this event was related to 
A

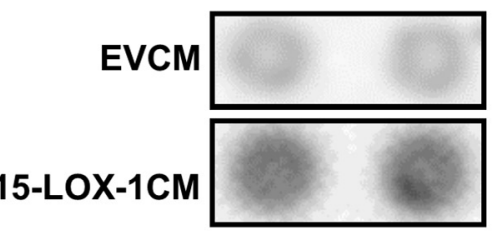

B

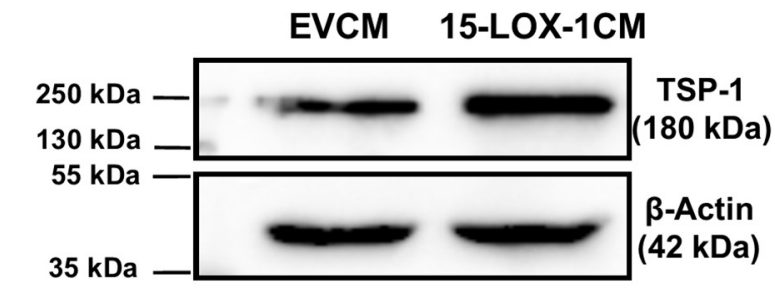

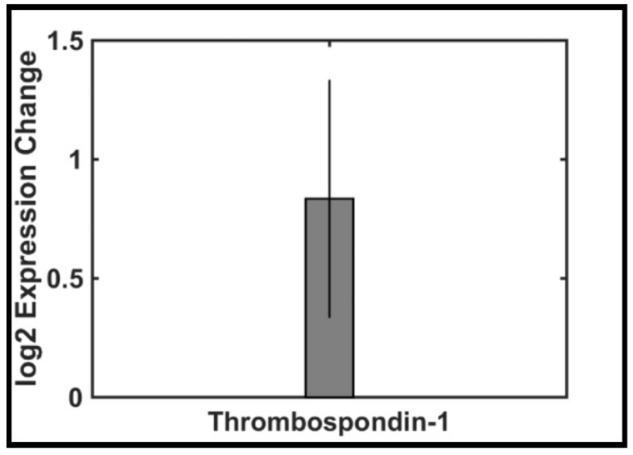

C
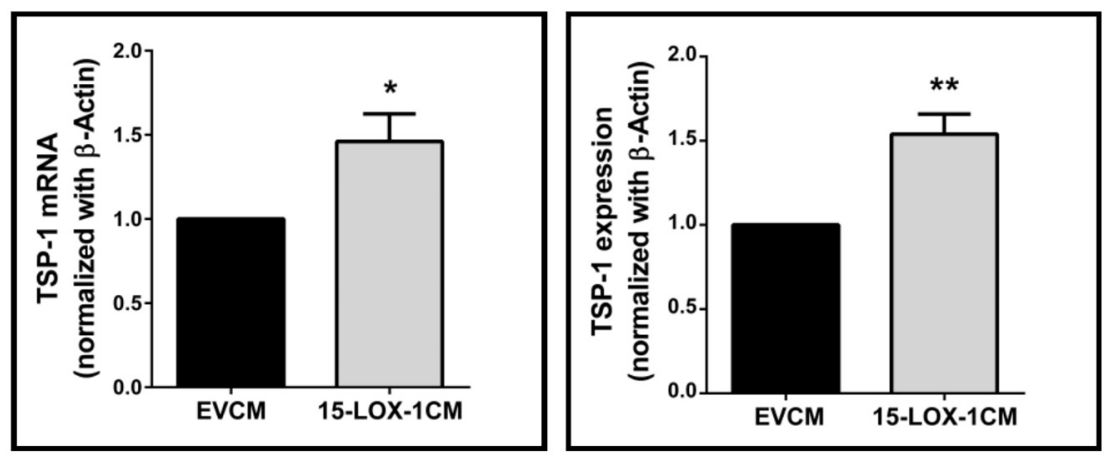

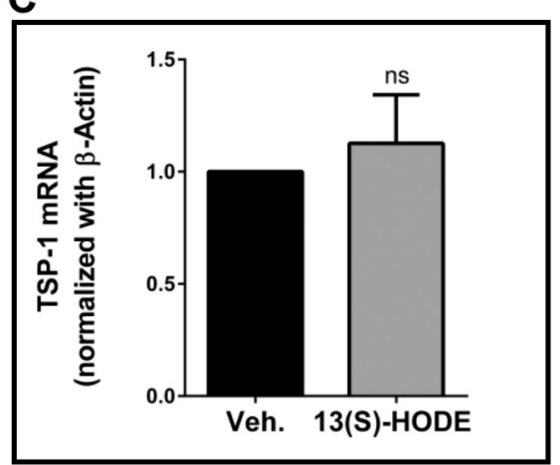

D

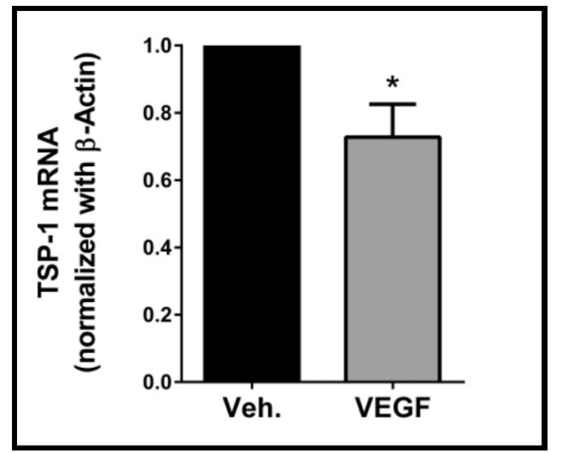

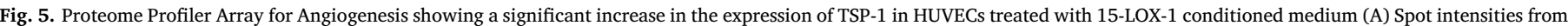

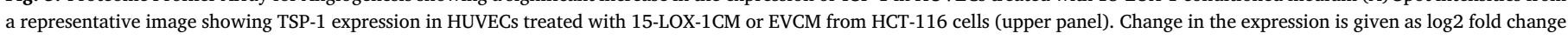

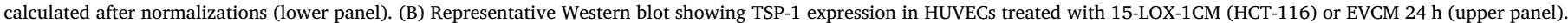

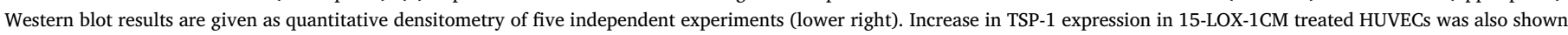

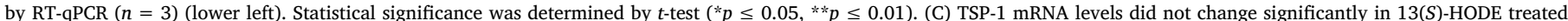
HUVECs $(n=3)$. (D) RT-qPCR shows decreased TSP-1 expression VEGF treated cells, respect to vehicle (Veh.) control $(n=4)$. $t$-Test was used for data analyses $(* p \leq 0.05)$.

the expression of TSP-1, we used bioinformatics tools to compare genes that were significantly downregulated in a microarray dataset from human microvascular endothelial cells (HMVECs) treated with a TSP-1 mimetic peptide [21] with genes that were significantly upregulated in human tumor associated endothelial cells from CRC [28]. A group of 54 intersect genes were significantly inversely correlated. STRING 10.0 analysis identified significant protein-protein interaction (PPI) enrichment $(p<0.00153$, data not shown) with one high density cluster (Supplementary Fig. 8). Functional enrichment in processes related to migration, morphogenesis and locomotion were identified.

\section{Discussion}

15-LOX-1 expression is widely suppressed in CRC, primarily through epigenetic mechanisms [2]. Re-expression of the protein in different cancer models has been shown to negate several hallmarks of cancer, including angiogenesis $[38,39]$. Here, we aimed to understand the mechanism through which 15-LOX-1 re-expression in CRC cell lines inhibited angiogenesis in endothelial cells.

New vessel formation is essential for a tumor to grow; limiting new vessel formation is widely considered to be an effective therapeutic tool in cancer [40]. The reduced transcription, expression and secretion of VEGF, a growth factor that is primarily responsible for angiogenesis, was observed with 15-LOX-1 overexpression in different cancer cell lines from different organs in the current study. This corroborates with data shown previously in both cancer $[39,41]$ and non-cancer models $[9,42]$. We did not observe any alterations in the activation (phosphorylation) of VEGF receptors in both HCT-116 and LNCaP cells expressing 15-LOX-1 (data not shown), indicating that rather than autocrine signaling, paracrine signaling between tumor cells and ECs in the tumor microenvironment may be functional in this model. Supporting this, we observed that 15-LOX-1CM could reduce sprouting in an ex vivo aorta ring assay as well as a HUVEC tube formation assay. Tumor vessels are known to be chaotic in their organization with tortuous structures that may be irregular, multilayered and poorly interconnected [7]. There has been a consensus in recent years that restoration of normal vascular architecture may enhance the success of chemotherapy [7]. We observed an obvious reduction in the formation of collateral vessels in the aortic rings and mesh structure in the tube formation assay in the presence of 15-LOX-1CM, indicating a potential reduction in the complexity of the structures.

Angiogenesis characteristically includes EC mitosis, extracellular matrix remodeling and sprouting [33]. We did not observe any changes in the proliferation or cell cycle distribution of HUVECs incubated with 15-LOX-1CM or EVCM. Significant inhibition, however, was observed in the motility of HUVECs incubated with 15-LOX-1CM. Mechanistically, this was through reduced expression of vimentin, a mesenchymal marker that is essential for cell motility [43]. 
A

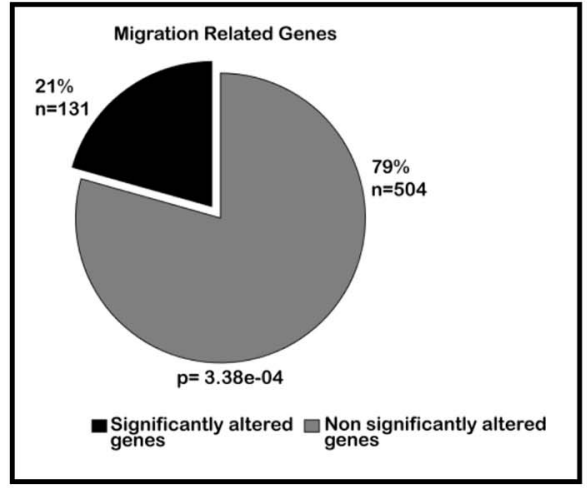

B

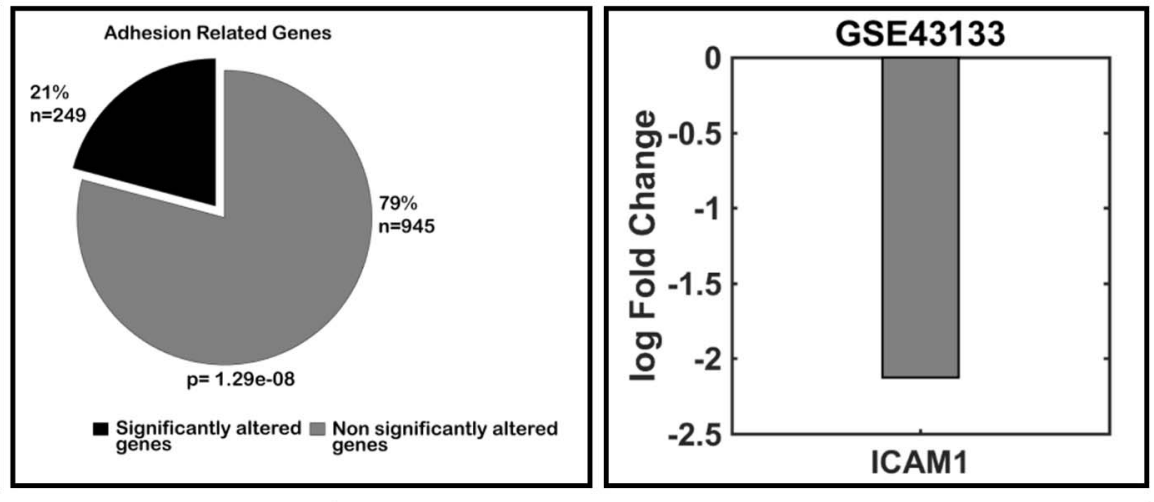

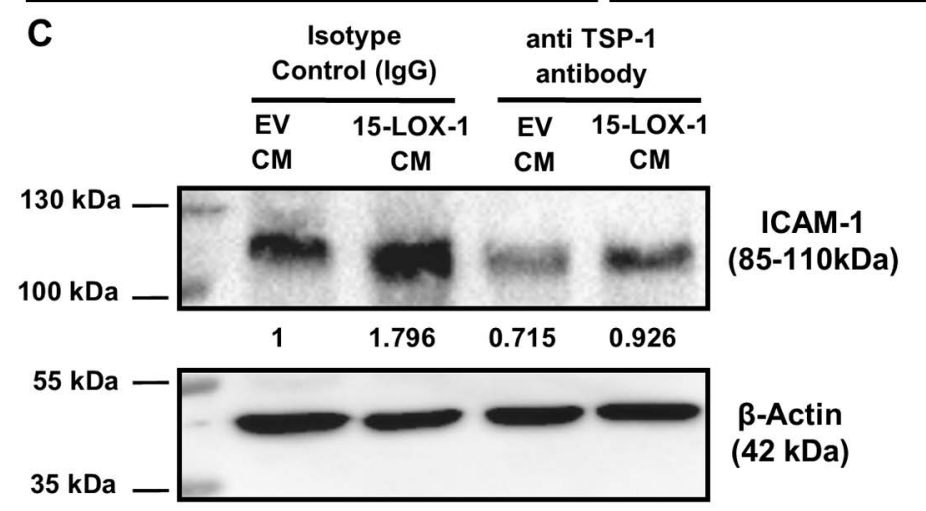

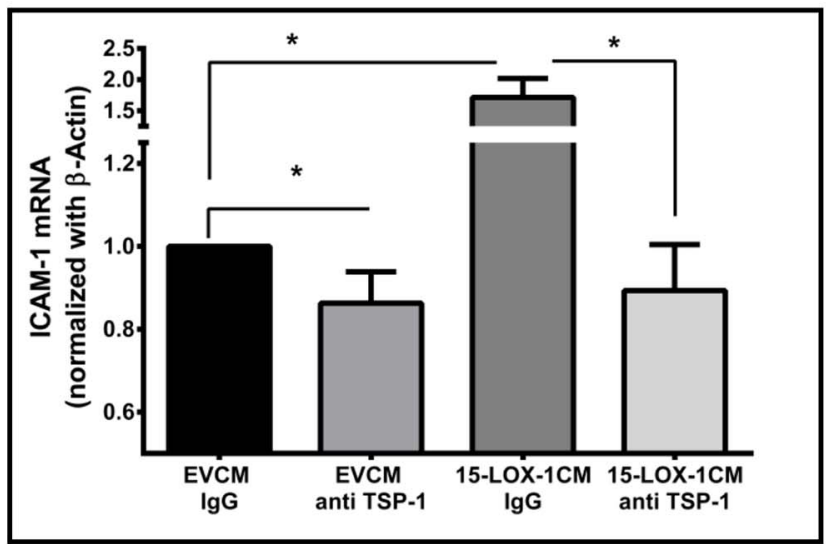

D

$\begin{array}{cc}\text { EVCM } & \text { EVCM } \\ \text { Isotype Control (IgG) } & \text { anti TSP-1 antibody }\end{array}$
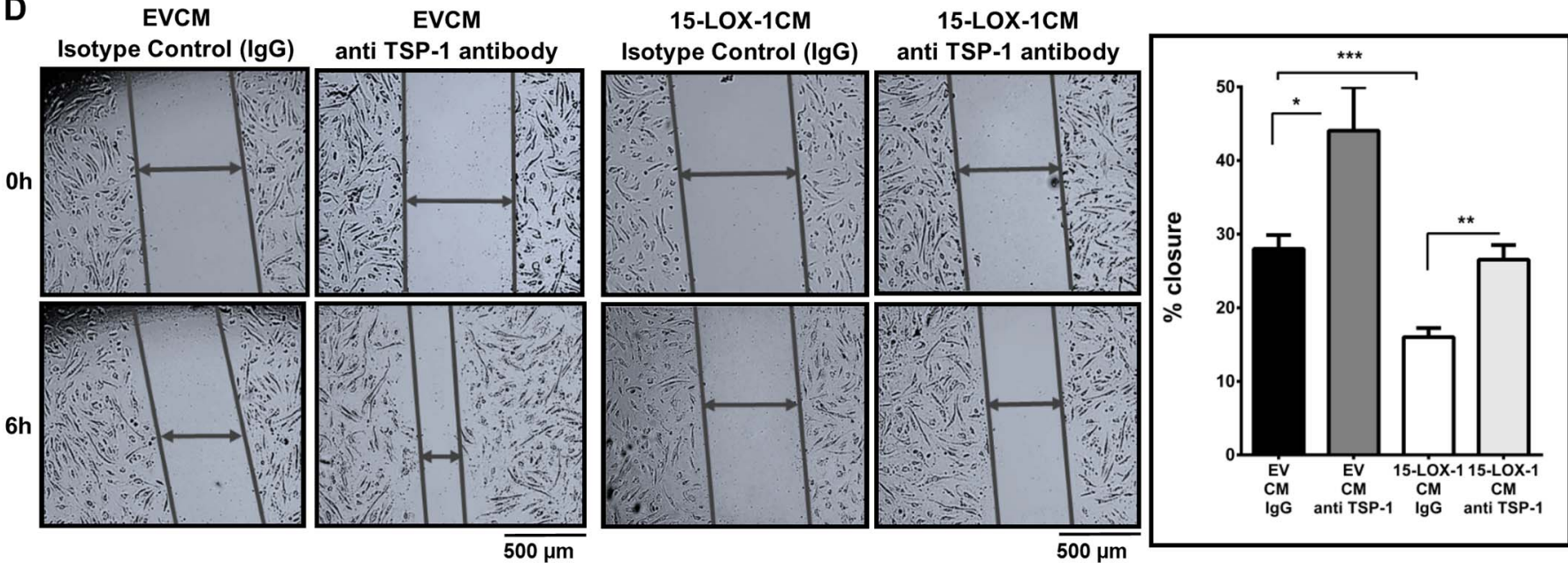

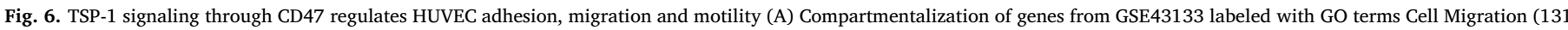

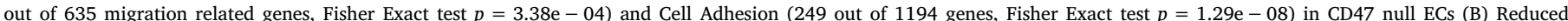

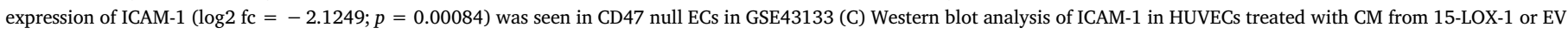

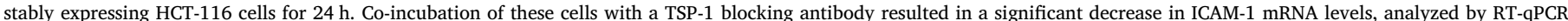

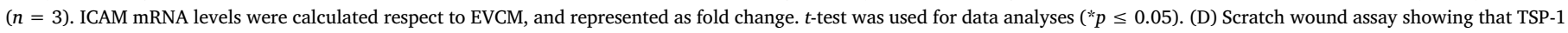

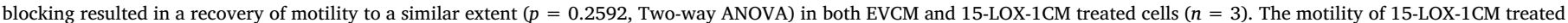
cells was in general low indicating that factors in addition to TSP-1 may also be functional. Statistical significance was determined by $t$-test $(* p \leq 0.05$, $* * p \leq 0.01, * * p \leq 0.001)$.

ECs interact with leukocytes with the help of cell adhesion molecules such as ICAM-1 [44]. The expression of cell adhesion molecules in tumor associated endothelial cells (TECs) have been previously shown to be reduced epigenetically through histone modifications via growth factors secreted by tumor cells [45]. This growth factor induced reduction in cell adhesion molecules, including ICAM-1, has been shown to reduce leukocyte infiltration in mouse ECs. This process, called endothelial cell anergy, results in reduced immune cell recruitment to the tumor microenvironment and enhanced tolerance [46]. We have observed a decrease in VEGF expression and secretion in epithelial cells expressing 15-LOX-1 and an increase in the expression of ICAM-1 in the HUVECs treated with 15-LOX-1CM from both colon and prostate cancer cell lines. These data imply that the 15-LOX-1CM treated HUVECs may be able to mitigate endothelial cell anergy.

We next examined whether the product of 15-LOX-1 enzymatic activity, 13(S)-HODE, can phenocopy the anti-angiogenic effects or 15LOX-1. We observed that ECs treated with 13(S)-HODE alone resulted in reduced cell motility and enhanced the expression of ICAM-1. This was expected, since enhanced expression of ICAM-1 in the presence of 13(S)-HODE has been reported in atherosclerotic lesions [47]. 


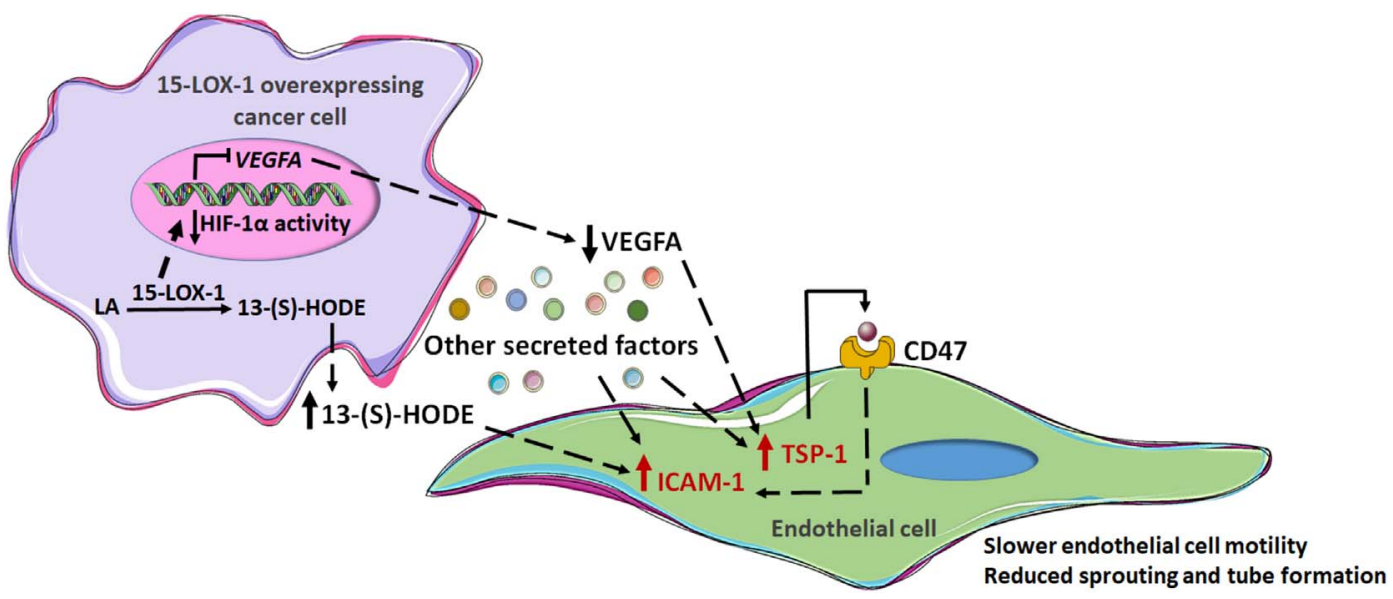

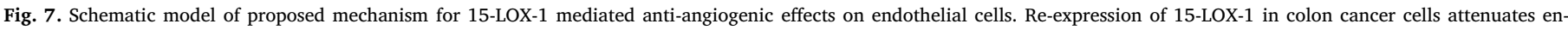

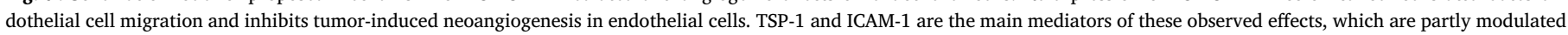
by $13(S)$-HODE, the main product of 15-LOX-1 metabolism.

However, more importantly, 13(S)-HODE did not affect the tube formation ability of HUVECs. This indicated the importance of a tumor microenvironment and presence of tumor associated factors in addition to13(S)-HODE in the current model.

An angiogenesis specific proteome array was therefore carried out and a significant and reproducible increase in TSP-1 expression was observed in HUVECs incubated with 15-LOX-1CM. The increase in TSP1 expression was confirmed at both mRNA and protein levels in HUVECs treated with 15-LOX-1CM from HCT-116 cells. TSP-1 is a member of the thrombospondin family and was the first endogenous anti-angiogenic protein discovered [35]. TSP-1 inhibits angiogenesis by vasoconstriction through the inhibition of nitric oxide signaling [48]. VEGF signaling has been shown to reduce the expression of TSP-1 through the upregulation of miR-17-92 cluster [49]; we observed that treatment of HUVECs with $100 \mathrm{ng} / \mathrm{ml}$ VEGF reduced the expression of TSP-1. It is therefore feasible that the reduced secretion of VEGF in the microenvironment of 15-LOX-1 expressing cancer cells may have contributed towards the enhanced expression of TSP-1 in ECs.

TSP-1 is known to signal in an autocrine manner through its receptor CD47 [50] and affect EC migration and adhesion [51]. Analysis of microarray data from CD47 null primary lung ECs indicated statistically significant alterations in GO terms related to migration and adhesion. ICAM-1 has been shown to be upregulated after the engagement of TSP-1 to CD47 in HUVECs [52] and human brain microvascular ECs [53]. Corroborating this, we observed a significant decrease in ICAM-1 in CD47 null mouse lung ECs from microarray data and an increase in the expression of ICAM-1 in HUVECs treated with 15-LOX-1CM, which was reversed when the cells were treated with a TSP-1 blocking antibody.

The TSP-1 interactome is enriched in ligands that are involved in extracellular matrix remodeling, cell- extracellular matrix interaction, cell-cell adhesion, motility and vascular normalization [52-54]. Pathway analysis of TECs in colorectal tumors compared to angiogenic (placental) and non-angiogenic ECs [55] indicated an enrichment of genes that are functional in cytoskeleton organization and extracellular matrix remodeling. This is highly reminiscent of the pathways altered in the current study. We therefore explored, using bioinformatics tools, whether TSP-1 signaling was involved in tumor vessel normalization. Common genes that were significantly downregulated in ECs treated with a TSP-1 mimetic and upregulated in CRC TECs were enriched for motility, further emphasizing the importance of TSP-1 mediated signaling in reducing EC motility, thereby enhancing normalization.

In conclusion, we have shown here that 15-LOX-1 expression in colon and prostate cancer cells leads to reduced angiogenesis. Alterations in pathways such as motility and adhesion were highly represented in our model and are likely to be among the major mechanisms for reduced angiogenesis observed when 15-LOX-1 is expressed in CRC cells. Mechanistically, we have shown for the first time that these changes could be mediated by an increase in the expression of both ICAM-1 and the anti-angiogenic protein TSP-1 (Fig. 7). The proteome array used in the current study is not all inclusive, involving only 55 proteins that play a role in angiogenesis. It is therefore likely that future studies will provide further mechanisms and pathways that may be activated as a result of 15-LOX-1 expression in CRC cells.

Supplementary data to this article can be found online at http://dx. doi.org/10.1016/j.cellsig.2017.07.022.

\section{Conflicts of interest}

None.

\section{Acknowledgements}

We thank Uddhav Kelavkar, Ilias Mylonis, Mayda Gürsel, Rafig Gurbanov and Feride Severcan for sharing resources, Sahika Koker for help with microscopy and Alper Duranel and members of the Banerjee Lab for useful discussions. We also thank Ebru Erbay for supervising the aorta isolation. The study was funded by TUBITAK projects 1135935 and 111 S308 to SB.

\section{References}

[1] M.J. Moussalli, Y. Wu, X. Zuo, X.L. Yang, I.I. Wistuba, M.G. Raso, J.S. Morris, J.L. Bowser, J.D. Minna, R. Lotan, I. Shureiqi, Mechanistic contribution of ubiquitous 15-lipoxygenase-1 expression loss in cancer cells to terminal cell differentiation evasion, Cancer Prev. Res. 4 (2011) 1961-1972.

[2] S.I.L. Lee, X. Zuo, I. Shureiqi, 15-Lipoxygenase-1 as a tumor suppressor gene in colon cancer: is the verdict in? Cancer Metastasis Rev. 30 (2011) 481-491.

[3] I. Çimen, S. Tunçay, S. Banerjee, 15-Lipoxygenase-1 expression suppresses the invasive properties of colorectal carcinoma cell lines HCT-116 and HT-29, Cancer Sci. 100 (2009) 2283-2291.

[4] S. Tunçer, S. Tunçay Çağatay, A.G. Keșküș, M. Çolakoğlu, Konu, S. Banerjee, Interplay between 15-lipoxygenase-1 and metastasis-associated antigen 1 in the metastatic potential of colorectal cancer, Cell Prolif. 49 (2016) 448-459.

[5] R. Tian, X. Zuo, J. Jaoude, F. Mao, J. Colby, I. Shureiqi, ALOX15 as a Suppressor of Inflammation and Cancer: Lost in the Link, Prostaglandins Other Lipid Mediat, (2017).

[6] K.M. Welch-Reardon, N. Wu, C.C.W. Hughes, A role for partial endothelial-mesenchymal transitions in angiogenesis? Arterioscler. Thromb. Vasc. Biol. 35 (2015) 303-308.

[7] M. Potente, H. Gerhardt, P. Carmeliet, Basic and therapeutic aspects of angiogenesis, Cell 146 (2011) 873-887.

[8] U.P. Kelavkar, J.B. Nixon, C. Cohen, D. Dillehay, T.E. Eling, K.F. Badr, Overexpression of 15-lipoxygenase-1 in PC-3 human prostate cancer cells increases tumorigenesis, Carcinogenesis 22 (2001) 1765-1773.

[9] H. Viita, J. Markkanen, E. Eriksson, M. Nurminen, K. Kinnunen, M. Babu, 
T. Heikura, S. Turpeinen, S. Laidinen, T. Takalo, S. Yla-Herttuala, 15-Lipoxygenase1 prevents vascular endothelial growth factor A- and placental growth factor-induced angiogenic effects in rabbit skeletal muscles via reduction in growth factor mRNA levels, NO bioactivity, and downregulation of VEGF receptor 2 expression, Circ. Res. 102 (2008) 177-184.

[10] Y. Yan, T. He, Y. Shen, X. Chen, B. Diao, Z. Li, Q. Liu, Y.Q. Xing, Adenoviral 15lipoxygenase-1 gene transfer inhibits hypoxia-induced proliferation of retinal microvascular endothelial cells in vitro, Int. J. Ophthalmol. (2012) 562-569.

[11] Z. Li, T. He, K. Du, Y.Q. Xing, Y.M. Run, Y. Yan, Y. Shen, Inhibition of oxygeninduced ischemic retinal neovascularization with adenoviral 15-lipoxygenase-1 gene transfer via up-regulation of PPAR- $\gamma$ and down-regulation of VEGFR-2 expression, PLoS One 9 (2014) e85824.

[12] Y. Wu, F. Mao, X. Zuo, M.J. Moussalli, E. Elias, W. Xu, I. Shureiqi, 15-LOX-1 suppression of hypoxia-induced metastatic phenotype and HIF-1 $\alpha$ expression in human colon cancer cells, Cancer Med. 3 (2014) 472-484.

[13] C.G. Kevil, A.W. Orr, W. Langston, K. Mickett, J. Murphy-Ullrich, R.P. Patel, D.F. Kucik, D.C. Bullard, Intercellular adhesion molecule-1 (ICAM-1) regulates endothelial cell motility through a nitric oxide-dependent pathway, J. Biol. Chem. 279 (2004) 19230-19238.

[14] P.R. Lawler, J. Lawler, Molecular basis for the regulation of angiogenesis by thrombospondin-1 and-2, Cold Spring Harb. Perspect. Med. 2 (2012) a006627.

[15] I. Cimen, E. Astarci, S. Banerjee, 15-lipoxygenase-1 exerts its tumor suppressive role by inhibiting nuclear factor-kappa B via activation of PPAR gamma, J. Cell. Biochem. 112 (2011) 2490-2501.

[16] M.W. Pfaffl, A new mathematical model for relative quantification in real-time RTPCR, Nucleic Acids Res. 29 (2001) 45e.

[17] S.A. Bustin, V. Benes, J.A. Garson, J. Hellemans, J. Huggett, M. Kubista, R. Mueller, T. Nolan, M.W. Pfaffl, G.L. Shipley, J. Vandesompele, C.T. Wittwer, The MIQE guidelines: minimum information for publication of quantitative real-time PCR experiments, Clin. Chem. 55 (2009) 611-622.

[18] T. Shibata, N. Akiyama, M. Noda, K. Sasai, M. Hiraoka, Enhancement of gene expression under hypoxic conditions using fragments of the human vascular endothelial growth factor and the erythropoietin genes, Int. J. Radiat. Oncol. Biol. Phys. 42 (1998) 913-916.

[19] M. Baker, S.D. Robinson, T. Lechertier, P.R. Barber, B. Tavora, G. D'Amico, D.T. Jones, B. Vojnovic, K. Hodivala-Dilke, Use of the mouse aortic ring assay to study angiogenesis, Nat. Protoc. 7 (2012) 89-104.

[20] S. Tuncay Cagatay, I. Cimen, B. Savas, S. Banerjee, MTA-1 expression is associated with metastasis and epithelial to mesenchymal transition in colorectal cancer cells, Tumour Biol. 34 (2013) 1189-1204.

[21] E.I. Cline, S. Bicciato, C. DiBello, M.W. Lingen, Prediction of in vivo synergistic activity of antiangiogenic compounds by gene expression profiling, Cancer Res. 62 (2002) 7143-7148.

[22] J. Wu, R.A. Irizarry, J. MacDonald, J. Gentry, Gcrma: Background Adjustment Using Sequence Information (R Package Version 2.22.0), (2016).

[23] M.E. Ritchie, B. Phipson, D. Wu, Y. Hu, C.W. Law, W. Shi, G.K. Smyth, Limma powers differential expression analyses for RNA-sequencing and microarray studies, Nucleic Acids Res. 43 (2015) e47.

[24] D. Smedley, S. Haider, S. Durinck, L. Pandini, P. Provero, J. Allen, O. Arnaiz, M. Awedh, R. Baldock, The BioMart community portal: an innovative alternative to large, centralized data repositories, Nucleic Acids Res. 43 (2015) W589-598.

[25] S. Carbon, A. Ireland, C.J. Mungall, S. Shu, B. Marshall, S. Lewis, J. Lomax, C. Mungall, B. Hitz, R. Balakrishnan, M. Dolan, V. Wood, E. Hong, P. Gaudet, AmiGO: online access to ontology and annotation data, Bioinformatics 25 (2009) 288-289.

[26] P.D. Thomas, M.J. Campbell, A. Kejariwal, H. Mi, B. Karlak, R. Daverman, K. Diemer, A. Muruganujan, A. Narechania, PANTHER: a library of protein families and subfamilies indexed by function, Genome Res. 13 (2003) 2129-2141.

[27] S. Kaur, D.R. Soto-Pantoja, E.V. Stein, C. Liu, A.G. Elkahloun, M.L. Pendrak, A. Nicolae, S.P. Singh, Z. Nie, D. Levens, J.S. Isenberg, D.D. Roberts, Thrombospondin-1 signaling through CD47 inhibits self-renewal by regulating cMyc and other stem cell transcription factors, Sci Rep 3 (2013) 1673.

[28] J. Wragg, J. Finnity, J. Anderson, H. Ferguson, E. Porfiri, R. Bhatt, P. Murray, V. Heath, R. Bicknell, MCAM and LAMA4 are highly enriched in tumor blood vessels of renal cell carcinoma and predict patient outcome, Cancer Res. 76 (2016) 2314-2326.

[29] D. Szklarczyk, A. Franceschini, S. Wyder, K. Forslund, D. Heller, J. Huerta-Cepas, M. Simonovic, A. Roth, A. Santos, K.P. Tsafou, M. Kuhn, P. Bork, L.J. Jensen, C. Von Mering, STRING v10: protein-protein interaction networks, integrated over the tree of life, Nucleic Acids Res. 43 (2015) D447-D452.

[30] N. Tuncbag, S.J.C. Gosline, A. Kedaigle, A.R. Soltis, A. Gitter, E. Fraenkel, Networkbased interpretation of diverse high-throughput datasets through the omics integrator software package, PLoS Comput. Biol. 12 (2016).
[31] K. Matsumoto, M. Ema, Roles of VEGF-A signalling in development, regeneration, and tumours, J. Biochem. 156 (2014) 1-10.

[32] H.L. Goel, A.M. Mercurio, VEGF targets the tumour cell, Nat. Rev. Cancer 13 (2013) 871-882.

[33] A.C. Dudley, Tumor endothelial cells, Cold Spring Harb, Perspect. Med. 2 (2012).

[34] P. Zhang, X. Xing, C. Hu, H. Yu, Q. Dong, G. Chang, S. Qin, J. Liu, D. Zhang, 15Lipoxygenase-1 is Involved in the Effects of Atorvastatin on Endothelial Dysfunction, Mediators Inflamm. (2016).

[35] B. Ren, K.O. Yee, J. Lawler, R. Khosravi-Far, Regulation of tumor angiogenesis by thrombospondin-1, Biochim. Biophys. Acta, Rev. Cancer 1765 (2006) 178-188.

[36] D.W. Dawson, S.F.A. Pearce, R. Zhong, R.L. Silverstein, W.A. Frazier, N.P. Bouck, CD36 mediates the in vitro inhibitory effects of thrombospondin-1 on endothelial cells, J. Cell Biol. 138 (1997) 707-717.

[37] L.Y. Chu, D.P. Ramakrishnan, R.L. Silverstein, Thrombospondin-1 modulates VEGF signaling via CD36 by recruiting SHP-1 to VEGFR2 complex in microvascular endothelial cells, Blood 122 (2013) 1822-1832.

[38] S. Tuncer, S. Banerjee, Eicosanoid pathway in colorectal cancer: recent updates, World J. Gastroenterol. 21 (2015) 11748-11766.

[39] Y. Wu, F. Mao, X. Zuo, M.J. Moussalli, E. Elias, W. Xu, I. Shureiqi, 15-LOX-1 suppression of hypoxia-induced metastatic phenotype and HIF-1 $\alpha$ expression in human colon cancer cells, Cancer Med. 3 (2014) 472-484.

[40] J. Folkman, Tumor angiogenesis: therapeutic implications, N. Engl. J. Med. 285 (1971) 1182-1186.

[41] H. Zhong, R. Wang, U. Kelavkar, C.Y. Wang, J. Simons, Enzyme 15-lipoxygenase 1 promotes hypoxia-inducible factor $1 \alpha$ turnover and reduces vascular endothelial growth factor expression: implications for angiogenesis, Cancer Med. 3 (2014) 514-525.

[42] Z. Li, T. He, K. Du, Y.Q. Xing, Y. Yan, Z. Chen, H. Zhang, Y. Shen, Overexpression of 15-lipoxygenase- 1 in oxygen-induced ischemic retinopathy inhibits retinal neovascularization via downregulation of vascular endothelial growth factor-a expression, Mol. Vis. 18 (2012) 2847-2859.

[43] J.M. Dave, K.J. Bayless, Vimentin as an integral regulator of cell adhesion and endothelial sprouting, Microcirculation 21 (2014) 333-344.

[44] D. Vestweber, How leukocytes cross the vascular endothelium, Nat. Rev. Immunol. 15 (2015) 692-704.

[45] D.M.E.I. Hellebrekers, K. Castermans, E. Viré, R.P.M. Dings, N.T.H. Hoebers, K.H. Mayo, M.G.A. Oude Egbrink, G. Molema, F. Fuks, M. Van Engeland, A.W. Griffioen, Epigenetic regulation of tumor endothelial cell anergy: silencing of intercellular adhesion molecule-1 by histone modifications, Cancer Res. 66 (2006) 10770-10777.

[46] A.E.M. Dirkx, Anti-angiogenesis therapy can overcome endothelial cell anergy and promote leukocyte-endothelium interactions and infiltration in tumors, FASEB J. 20 (2006) 621-630.

[47] V. Vangaveti, B.T. Baune, R.L. Kennedy, Review: hydroxyoctadecadienoic acids: novel regulators of macrophage differentiation and atherogenesis, Ther. Adv. Endocrinol. Metab. 1 (2010) 51-60.

[48] J.S. Isenberg, G. Martin-Manso, J.B. Maxhimer, D.D. Roberts, Regulation of nitric oxide signalling by thrombospondin 1: implications for anti-angiogenic therapies, Nat. Rev. Cancer 9 (2009) 182-194.

[49] Y. Suarez, C. Fernandez-Hernando, J. Yu, S.A. Gerber, K.D. Harrison, J.S. Pober, M.L. Iruela-Arispe, M. Merkenschlager, W.C. Sessa, Dicer-dependent endothelial microRNAs are necessary for postnatal angiogenesis, Proc. Natl. Acad. Sci. 105 (2008) 14082-14087.

[50] S.S. Li, Z. Liu, M. Uzunel, K.G. Sundqvist, Endogenous thrombospondin-1 is a cellsurface ligand for regulation of integrin-dependent T-lymphocyte adhesion, Blood 108 (2006) 3112-3120.

[51] E. Sick, A. Jeanne, C. Schneider, S. Dedieu, K. Takeda, L. Martiny, CD47 update: a multifaceted actor in the tumour microenvironment of potential therapeutic interest, Br. J. Pharmacol. 167 (2012) 1415-1430.

[52] N.V. Narizhneva, O.V. Razorenova, E.A. Podrez, J. Chen, U.M. Chandrasekharan, P.E. DiCorleto, E.F. Plow, E.J. Topol, T.V. Byzova, Thrombospondin-1 up-regulates expression of cell adhesion molecules and promotes monocyte binding to endothelium, FASEB J. 19 (2005) 1158-1160.

[53] C. Xing, S. Lee, J.K. Woo, H. Wang, Y.G. Yang, M. Ning, X. Wang, E.H. Lo, Neurovascular effects of CD47 signaling: promotion of cell death, inflammation, and suppression of angiogenesis in brain endothelial cells in vitro, J. Neurosci. Res. 87 (2009) 2571-2577.

[54] A. Resovi, D. Pinessi, G. Chiorino, G. Taraboletti, Current understanding of the thrombospondin-1 interactome, Matrix Biol. 37 (2014) 83-91.

[55] J.R. Van Beijnum, R.P. Dings, E. Van Der Linden, B.M.M. Zwaans, F.C.S. Ramaekers, K.H. Mayo, A.W. Griffioen, Gene expression of tumor angiogenesis dissected: specific targeting of colon cancer angiogenic vasculature, Blood 108 (2006) 2339-2348. 